\title{
Original Research Article: Clinical Profile and Outcome of Patients with Acute Encephalitis Syndrome in a Tertiary Care Hospital, Assam, India.
}

\author{
Dr. Anuradha Deuri ${ }^{1}$, Dr. D. Kalita ${ }^{2}$ \\ ${ }^{I}$ Associate Professor, Department Of Medicine, F A A Medical College and Hospital, Barpeta, Assam.,India. \\ ${ }^{2}$ Assistant Professor of Pediatrics, Gauhati Medical College, Guwahati, Assam., India.
}

\begin{abstract}
:
Background: Acute Encephalitis Syndrome (AES) is a leading cause of mortality and morbidity. Japanese encephalitis (J.E.) is the most prevalent mosquito borne viral encephalitis, occurring in Japan, China, Southeast Asia and India, affecting around 50,000 people and 15,000 deaths annually (4, 5). Acute Encephalitis Syndrome (AES) is a major health problem in the state of Assam. In the state of Assam, several districts of upper Assam, have been experiencing recurrent episodes of $A E S$ ( $J E$ and non $J E$ ) with different magnitudes in the post monsoon period leading to many deaths and greater numbers as disabled (7). Under this backdrop, the present study was undertaken to know the epidemiology, clinical profile and outcome of AES patients.

Methods: The present study was carried out in department of Medicine, F.A.A. Medical College and hospital, Barpeta, Assam. F.A.A Medical College hospital is a tertiary care hospital, located in rural Assam. A total number of 60 patients with acute encephalitis syndrome admitted in Medicine ward over a period of 2 years from February 2014 to January 2016, were included in this study. This was a retrospective study. We collected the bed head tickets and all investigation reports (medical files) of all AES patients from medical record department of the hospital. Relevant data of the AES cases were collected. Next, data were analyzed and interpreted accordingly.

Results: A total number of 60 AES cases were included in the present study. Out of 60 AES patients, majority $37(61.7 \%)$ of them were below 30 years of age. Male were 38 (63.3\%) and female cases were 22(36.7\%). Most of the cases were seen in rural area 56 (93.3\%) suggesting that AES mainly affect rural population of Assam .Peak incidence was seen in monsoon and post monsoon period. The most common presenting clinical manifestations were fever with altered sensorium (100\%) followed by headache (93.3\%) and vomiting (58.3\%). Seizure was seen in 26 (43.3\%) cases. The most common outcome of AES cases was full recovery, seen in 38 cases (65.5\%). Partial recovery i.e. with neurological sequelae was seen in 11 (18.9\%) cases. The mortality rate of AES was $15.5 \%$ in this study.

Conclusion: Acute viral (Non J E) encephalitis still remains most common cause of AES followed by JE positive encephalitis. Immunization programme with $J E$ vaccine is an important measure for prevention of the diseases. Proper supporative care and timely intervention in intensive care unit will save many lives. Physiotherapy and family support will improve long term outcome of AES cases with neurological sequelae. Case reporting and appropriate workup of all cases would strengthen the AES surveillance and go a long way in bringing down the morbidity and mortality associated with AES.
\end{abstract}

Keywords: acute encephalitis syndrome (AES), fever with altered sensorium, seizure, J.E.vaccine, neurological sequelae, mortality.

\section{Introduction}

Encephalitis is defined as an acute inflammatory process of the brain parenchyma with dysfunction of the brain. The disease is most commonly caused by viral infection. Acute viral encephalitis is the most common cause of acute encephalitis syndrome (1). Acute Encephalitis Syndrome (AES) including Japanese Encephalitis (JE) is a group of clinically similar neurologic manifestation caused by several different viruses, bacteria, fungus, parasites, spirochetes etc. There is seasonal and geographical variation in the causative organism. The outbreak of JE usually occurs with the monsoon and post monsoon period as the density of mosquitoes increases during this season. On the other hand, encephalitis due to other viruses specially entero-viruses occurs throughout the year as it is a water borne disease. The morbidity and mortality is very high among various viral encephalitis specially in JE or enterovirus encephalitis in various parts of India (2). Children and young adult remain the main victims of AES. The incidence of viral encephalitis is $3.5-7.4$ per 1,00,000 population per year ( 3 ). It may be sporadic like herpes 
Original, Research Article Clinical Profile And Outcome Of Patients With Acute Encephalitis...

simplex encephalitis ,or epidemic such as Japanese B encephalitis (J E ). There is a considerable variation in the incidence and severity of sequelae in patients surviving viral encephalitis. The severity of sequelae is directly related to the age of the patient and the level of consciousness at the time of initiation of therapy. Young patient < 30 years of age with good neurologic function at initiation of therapy did substantially better in respect to survival and sequelae compared with their older counter part(1). In treatment, specific antiviral therapy should be initiated when appropriate but intensive supporative care is the mainstay to avoid neurological sequelae. $\mathrm{J} E$ is the most prevalent mosquito borne viral encephalitis , occurring in Japan, China, Southeast Asia and India, affecting around 50,000 people and 15,000 deaths annually $(4,5)$. In India, nearly all states have reported JE cases except that of Jammu \& Kashmir, Himachal Pradesh, and Uttaranchal (6).

Acute Encephalitis Syndrome (AES) is a major health problem in the state of Assam as it affects thousands of patient every year. In the state of Assam, particularly several districts of upper Assam , namely Dibrugarh, Jorhat, Tinsukia, Sivasagar, Golaghat and North lakhimpur have been experiencing recurrent episodes of AES ( $\mathrm{J} \mathrm{E}$ and non JE ) with different magnitudes in the post monsoon period from July to October, leading to many deaths and greater numbers as disabled (7). But, this disease is now, rapidly spreading into the various districts of lower Assam ,namely Kamrup (rural), Barpeta, Nalbari, Bongaigaon, Baska, Chirang and Kokrajhar with high morbidity and mortality. Many studies were carried out by various workers and agencies to study the epidemiological factor, clinical profile and outcome etc , mainly in the upper Assam in the past 15 years. But, very little data is available in this respect from the newly affected districts of lower Assam. Under this backdrop, the present study was undertaken in a tertiary care hospital, which is located in lower Assam and mainly caters services to people of these newly affected districts. The objectives of the present study were to know better the epidemiology, clinical profile and outcome among AES patients admitted in this hospital.

\section{Methods}

The present study was carried out in department of Medicine , F.A.A. Medical College and hospital ,Barpeta, Assam. F.A.A Medical College hospital is a tertiary care hospital ,located in rural Assam and mainly cater health care services in 6 to 7 districts namely Barpeta, Nalbari, Baska, Chirang, Bongaigaon, Dhubri and kokrajhar in lower Assam. These are the newly affected districts with AES cases in Assam. Most of the A ES cases from these districts were referred to this hospital for better management for intensive care facility, neuroimaging, advanced serological test ,CSF analysis, haematological tests and metabolic panel studies. A total number of 60 patients with acute encephalitis syndrome admitted in Medicine ward over a period of 2 years from February 2014 to January 2016, were included in this study. This was a retrospective study. We collected the bed head tickets and investigation reports (medical files) of all AES patients from medical record department of the hospital after taking permission from the concern hospital authority. All the medical files were evaluated thoroughly and relevant data of the AES cases were collected. WHO case definition of AES cases was adopted for selection of cases under this study. Case definition of Acute Encephalitis Syndrome (AES) Clinically, a case of AES is defined as a person of any age, at any time of year with the acute onset of fever and a change in mental status (including symptoms such as confusion, disorientation, coma, or inability to talk) AND/OR new onset of seizures (excluding simple febrile seizures). Other early clinical findings may include an increase in irritability, somnolence or abnormal behavior, greater than that seen with usual febrile illness $(2,16)$. Those patients with laboratory findings point to other diagnosis were excluded from the study. Patients were excluded if they had severe infection other than in the central nervous system, malignancy, brain infarction or cerebral hemorrhage and malaria. Further, patients with a diagnosis of delirium or encephalopathy secondary to metabolic causes were excluded from this study. Next, collected data were analyzed and interpreted accordingly. Two patients who left hospital against medical advice were disqualified from the outcome analysis.

\section{Results}

Table- 1 shows demographic profile of acute encephalitis syndrome (AES) patients.( $n=60$ )

\begin{tabular}{|l|l|l|l|}
\hline Sl. No. & Parameter & Number of patients & percentage \\
\hline Age: & 18 to 30 years & 37 & $61.7 \%$ \\
\hline & 30 to 50 years & 21 & $35 \%$ \\
\hline & More than 5o years & 2 & $3.3 \%$ \\
\hline Sex & Male & 38 & $63.3 \%$ \\
\hline & Female & 22 & $36.7 \%$ \\
\hline Settings & Urban & 4 & $6.7 \%$ \\
\hline & Rural & 56 & $93.3 \%$ \\
\hline
\end{tabular}


Original, Research Article Clinical Profile And Outcome Of Patients With Acute Encephalitis...

A total number of 60 AES cases were included in the present study. Out of 60 AES patients, majority $37(61.7 \%)$ of them were below 30 years of age. Male were $38(63.3 \%)$ and female cases were 22(36.7\%). Most of the cases were seen in rural area 56 (93.3\%) suggesting that AES mainly affect rural population of Assam . Further, In month wise distribution of cases , $47(78.3 \%)$ pattients were observed in the month of July ,August , September and October suggesting peak incidence in monsoon and post monsoon period.

Table-2 showing presenting clinical manifestations of acute encephalitis syndrome (AES) patients.( $n=60)$

\begin{tabular}{|l|l|l|l|}
\hline Sl. No. & Clinical manifestation & Number of patients & Percentage \\
\hline 1 & History of fever & 60 & $100 \%$ \\
\hline 2 & Headache & 56 & $93.3 \%$ \\
\hline 3 & Altered sensorium & 60 & $100 \%$ \\
\hline 4 & Confusion & 48 & $80 \%$ \\
\hline 5 & Behavioral abnormality & 7 & $11.7 \%$ \\
\hline 6 & Coma & 5 & $8.3 \%$ \\
\hline 7 & Vomiting & 35 & $58.3 \%$ \\
\hline 8 & Seizure & 26 & 43.3 \\
\hline a & Focal seizure & 4 & $6.7 \%$ \\
\hline b & Generalized seizure & 22 & $36.7 \%$ \\
\hline 9 & Breathlessness & 9 & $15 \%$ \\
\hline 10 & Abdominal pain with loose stool & 11 & $18.3 \%$ \\
\hline 11 & Swelling of body & 4 & $6.7 \%$ \\
\hline
\end{tabular}

The most common presenting clinical manifestations were fever with altered sensorium (100\%) followed by headache $(93.3 \%)$ and vomiting $(58.3 \%)$. Seizure was seen in $26(43.3 \%)$ cases.

Table-3 showing clinical findings of general examination of acute encephalitis syndrome (AES) patients.( $\mathrm{n}=60$ )

\begin{tabular}{|l|l|l|l|}
\hline Sl. No. & clinical examination findings & Number of patients & Percentage \\
\hline 1 & Temperature $>100 \mathrm{~F}$ & 41 & $68.3 \%$ \\
\hline 2 & Tachycardia & 18 & $30 \%$ \\
\hline 3 & Bradycardia & 2 & $3.3 \%$ \\
\hline 4 & Tachypnoea & 16 & $26.6 \%$ \\
\hline 5 & Pallor & 14 & $23.3 \%$ \\
\hline 6 & Icterus & 2 & $3.3 \%$ \\
\hline 7 & Lymphadenopathy & 2 & $3.3 \%$ \\
\hline 8 & Edema & 4 & $6.7 \%$ \\
\hline 9 & Glasgow coma scale $<8$ & 24 & $40 \%$ \\
\hline
\end{tabular}

On general examination findings, documented fever $>100 \mathrm{~F}$ was seen in $41(68.3 \%)$, Tachycardia (30\%) and Tachypnoea (26.6\%) cases. 24 AES cases (40\%) had glassgow coma scale (GCS) within 3to 8. Out of 60 AES cases, 58 patients were presented to the hospital between 1 to 10 days of onset of symptoms.

Table-4 showing clinical findings of systemic examination of acute encephalitis syndrome (AES) patients. $(n=60)$

\begin{tabular}{|l|l|l|l|}
\hline A & Abdominal examination & Number of patients & Percentage \\
\hline 1 & Hepatomegly & 9 & $15 \%$ \\
\hline 2 & Splenomegaly & 4 & $6.7 \%$ \\
\hline 3 & Hepatosplenomegaly & 6 & $10 \%$ \\
\hline 4 & Ascites & 2 & $3.3 \%$ \\
\hline B & Respiratory system & & \\
\hline 1 & Bronchial breath sound & 1 & $1.7 \%$ \\
\hline 2 & Adventitious sound(crepitation \& rhonchi) & 12 & $20 \%$ \\
\hline 3 & Respiratory distress & 5 & $8.3 \%$ \\
\hline C & Cardiovascular system & & \\
\hline 1 & Muffled heart sound & 2 & $3.3 \%$ \\
\hline 2 & Gallop rhythm & 2 & $3.3 \%$ \\
\hline 3 & Murmur & 1 & $1.7 \%$ \\
\hline D & Central nervous system & & \\
\hline 1 & Signs of meningeal irritation & 5 & $8.3 \%$ \\
\hline 2 & Hemiparesis & 6 & $10 \%$ \\
\hline 3 & Aphasia & 4 & $6.7 \%$ \\
\hline 4 & Ocular palsy & 3 & $5 \%$ \\
\hline 5 & Facial palsy & 2 & $3.3 \%$ \\
\hline
\end{tabular}


Original, Research Article Clinical Profile And Outcome Of Patients With Acute Encephalitis...

On evaluation of systemic examination findings, hepatomegaly was noted in 9 cases (15\%), respiratory distress 5 cases, gallop rhythm 2 cases, Signs of meningeal irritation 5 cases, Hemiparesis 6 cases and ocular palsy in 3 patients.

Table-5 showing complications developed during hospital stay of acute encephalitis syndrome (AES) patients.

\begin{tabular}{|l|l|l|l|}
\hline Sl. No. & Complication & Number of patients & Percentage \\
\hline 1 & Aspiration pneumonia & 6 & $10 \%$ \\
\hline 2 & Respiratory failure & 5 & $8.3 \%$ \\
\hline 3 & Psychosis & 5 & $8.3 \%$ \\
\hline 4 & Peripheral circulatory failure & 4 & $6.7 \%$ \\
\hline 5 & Upper G I bleeding & 1 & $1.7 \%$ \\
\hline 6 & Bedsore & 3 & $5 \%$ \\
\hline 7 & Sepsis & 1 & $1.7 \%$ \\
\hline
\end{tabular}

The common complications developed during hospital stay were Aspiration pneumonia 6 cases (10\%) followed by Respiratory failure $5(8.3 \%)$ and Psychosis 5 (8.3\%) patients.

After detailed evaluation of all laboratory investigation findings, it was found that 13 cases $(21.7 \%)$ were JE positive and rest 47 ( $78.3 \%$ ) cases were non JE encephalitis.

Table-6 showing outcome of acute encephalitis syndrome (AES) patients at the time discharge from hospital( $\mathrm{n}=58$ )

( 2 AES patients left hospital against medical advice, hence not included in outcome analysis

\begin{tabular}{|l|l|l|l|}
\hline S1. No. & Outcome & Number of patients & percentage \\
\hline 1 & Recovered completely & 38 & $65.5 \%$ \\
\hline 2 & Recovered with neurological sequelae & 11 & $18.9 \%$ \\
\hline 3 & Death & 9 & $15.5 \%$ \\
\hline
\end{tabular}

On evaluation of outcome of AES cases, It was seen that outcome at discharge was recorded in 58 cases as 2 patients left against medical advice. The most common outcome of AES cases was full recovery, seen in 38 cases (65.5\%). Partial recovery i.e. with neurological sequelae was seen in 11 (18.9\%) cases. Of all AES cases, 9 (15.5\%) patients were expired in the hospital. Therefore, the case fatality rate of AES was $15.5 \%$ in this study.

\section{Discussion}

The present study retrospectively analyzed data of 60 patients with AES admitted in a tertiary care hospital. This study reveals that majority (61.7\%) of AES cases belonged to less than 30 years of age, suggesting the diseases has higher incidence in younger population. This observation was similar to many studies like ,one study done by kumar R et al(2014) found (54.6\%) in Uttar Pradesh,India and another study done by jain P et al (2014)in India. $(8,9)$. It could be explained by younger patients were more affected probably because of lack of cumulative immunity due to natural infection. A male preponderance was seen in our study which was similar with various earlier studies $(8,9,10,15)$. It could be explained probably due to males are exposed more in outdoor activities in regards to females who mostly stay indoors. Our study also indicates that patients mostly affected were from rural areas of Assam (93.4\%) and belong to low socioeconomic group. Similar observations were made by Kakoti G et al (2013) $90 \%$ in upper Assam and Kumar R at el (2006) in Uttar Pradesh, India (10,11). In month wise distribution of cases , (78.3\%) patients were observed in the month of July ,August, September and October suggesting peak incidence in monsoon and post monsoon period. Many workers reported about this peak incidence in their studies. $(8,9,10,15)$. Further, we found that most of the patients were farmers by occupation. Farmers used to go to paddy field during this season regularly as this is the best time for cultivation in Assam. This may be due to several favorable epidemiological factors like presence of water logged paddy field supporting profuse breeding of vector mosquitoes, birds like herons are common in the paddy fields during this season and piggeries are more common in Assam and located near house hold campus of the villager. Again, villagers were not using mosquito net regularly at night and at the same time they do not wear proper covered clothing while working in paddy field . Hence, they are more prone to have mosquito bites.

The present study reveals that the most common presenting clinical manifestations were fever with altered sensorium (100\%) followed by headache (93.3\%) and vomiting (58.3\%). Seizure was seen in (43.3\%) cases. Similar observations were made by different researchers in earlier studies $(8,9,10,12)$.

In our study, after detailed evaluation of all laboratory investigation reports, it was found that the most common cause of AES was acute ( Non JE) encephalitis (78.3\%). It was followed by acute JE positive (21.7\%) encephalitis. 
Original, Research Article Clinical Profile And Outcome Of Patients With Acute Encephalitis...

It was comparable to many earlier studies done in India. Kumar R et al (2014) reported (29.2\%) JE positive and another study done by Kakoti $\mathrm{G}$ et al (2013) found $30 \% \mathrm{JE}$ positive encephalitis $(8,10)$. This clearly shows that the most common cause of AES was acute viral (Non JE) encephalitis at the community level. Hence, we have to adopt appropriate measure to control these (Non J E) cases to bring down the morbidity and mortality of AES. This calls for the further studies with large sample size to find out the exact etiological agent for the Non JE AES and this will help in planning the preventive strategies.

In India, Immunization programme, with $\mathrm{JE}$ vaccine has been initiated in the different endemic states to combat JE encephalitis. Currently in Assam, JE vaccination with live vaccine (SA-14-14-2) has been included in routine immunization programme as per National Immunization Schedule (NIS). Previously to clear the backlog in children 1-15 years of age mass vaccination programme was conducted in $11 \mathrm{JE}$ endemic districts of Assam in a phase wise manner since May 2006 (10).

The present study reviews the outcome of AES cases. The most common outcome of AES cases was full recovery, seen in $(65.5 \%)$. Partial recovery i.e. with neurological sequelae were seen in (18.9\%) cases. This was similar as previous studies done by Kumar R et al (2014) and Kakoti G et al (2013) (8, 10). The common neurological sequelae were cognitive impairment, hemiparesis, psychosis and extrapyramidal symptoms. In our study, GCS score below 8, concurrent seizure and coma at the time of admission were found to be poor prognostic factor and associated with increased mortality. Similar observations were made by various workers in their earlier studies, Ooi MH et al(2008) in Malaysia, Y C Wu et al(1999) in Taiwan $(13,14)$.

The present study demonstrates the mortality rate of AES was $15.5 \%$. Again, mortality was associated with GCS within 3to 8. The mortality rate of AES varies from 10 to $40 \%$ as described in different studies $(8,9$ ,10,15).

\section{Conclusion}

The present study summarises the epidemiological factors, clinical profile and outcome of AES cases. Acute viral (Non J E) encephalitis still remains most common cause of AES followed by JE positive encephalitis. Immunization programme with $\mathrm{J} \mathrm{E}$ vaccine is an important measure for prevention of Japanese encephalitis. Health education and awareness programme among public regarding use of mosquito net, proper clothing etc to prevent mosquito bites is of long term need. Moreover, continuation of JE vaccination of children in routine immunization in these JE endemic districts of Assam should be a public health priority. Proper supporative care and timely intervention in intensive care unit will save many lives. Physiotherapy and family support will improve long term outcome of AES cases with neurological sequelae. However, further studies with large sample size is needed to find out the exact etiological agent for the Non JE AES and this will help in planning the preventive strategies in near future. Case reporting and appropriate workup of all cases would strengthen the AES surveillance and go a long way in bringing down the morbidity and mortality associated with AES.

Limitation: Sample size was small so result cannot be generalized to general population as data was collected from a single hospital. Further, studies with large sample size is needed in Assam.

Funding: No funding sources.

Conflict of interest: None stated.

Authors contribution: Anuradha Deuri had collected, analyzed and interpreted data, written manuscript.

D. Kalita had analyzed and interpreted data, written manuscript.

\section{References}

[1]. Dan L. longo, Dennis L. Kasper, J Larry Jameson, Anthony S Fauci,Stephen L hauser, Joseph Loscalzo. : Harrison's Principles of Internal Medicine: Mc Graw Hill,2012: $18^{\text {th }}$ edition, volume 2; pages 3421-3426.

[2]. Resource manual, National vector borne disease control programme, in India: Directorate General of Health services, 2014: Govt. of India.

[3]. Gendleman HE, Persidsky Y. Infections of the Nervous system. Lancet Neurol 2005; 4: 12-13.

[4]. Sejvar JJ. The evolving epidemiology of viral encephalitis. Curr opin Neurol 2006; 19: 350-357.

[5]. Panagariya A, Jam RS, Gupta S, Garg A, Sureka RK, Mathur V. Herpes simplex encephalitis in North West India. Neurol India. 2001 ; 49: 360-365.

[6]. N. Arunachalam, R. Rajendran, P. P. Samual et al., "Studies on Japanese encephalitis in Kurnool district, Andhra Pradesh," CRME Annual Report. 
[7]. P. Dutta, S. A. Khan, A. M. Khan, J. Borah, C. K. Sarmah,and J. Mahanta, "The effect of Insecticide-Treated Mosquito Nets (ITMNs) on Japanese encephalitis virus seroconversion in pigs and humans," American Journal of Tropical Medicine and Hygiene, vol. 84, no. 3, pp. 466-472, 2011.

[8]. Kumar R, Bhushan M, Nigam P: Pattern of infections in adult patients presenting as acute encephalitis syndrome(AES). International journal of Medical science education.: vol 1, issue 4; oct-Dec, 2014.

[9]. Jain P at al. Epidemiology \& aetiology of AES in north India. Jpn J Infect dis 2014; 167: 197-203.

[10]. Kakoti G, Dutta P,Das B R,Borah J,Mahanta J: Clinical profile and outcome of Japanese encephalitis in children admitted with acute encephalitic syndrome; Hindawi publishing corporation: Biomed research international: vol 2013, article id 152656, 5 pages.

[11]. R. Kumar, P. Tripathi, S. Singh, and G. Bannerji, "Clinical features in children hospitalized during the 2005 epidemic of Japanese encephalitis in Uttar Pradesh, India," Clinical Infectious Diseases, vol. 43, no. 2, pp. 123-131, 2006.

[12]. K.-M. Chen, H.-C. Tsai, C.-L. Sy et al., "Clinical manifestations of Japanese encephalitis in southern Taiwan," Journal of Microbiology, Immunology and Infection, vol. 42, no. 4, pp. 296-302, 2009.

[13]. Ooi MH, Lewthwaite $\mathrm{P}$, Lai BF et al (2008) The epidemiology, clinical features, and long-term prognosis of Japanese encephalitis in central Sarawak, Malaysia, 1997-2005. J Clin Infect Dis 47(4):458-468.

[14]. Y. C. Wu, Y. S. Huang, L. J. Chien et al., "The epidemiology of Japanese encephalitis inTaiwan during 1996-1997," American Journal of Tropical Medicine and Hygiene, vol. 61, pp.78-84,1999.

[15]. 15) De .S, Samanta S, et al: Clinical profile and outcome of children admitted with acute encephalitis syndrome in a tertiary care hospital in West Bengal , India. IOSR journal of Dental and Medical Sciences; volume 14,issue 11, ver.v11 (Nov 2015),PP 08-12

[16]. T. Solomon, T. T. Thao, P. Lewthwaite et al., "A cohort study to assess the new WHO Japanese encephalitis surveillance standards,"Bulletin of the World Health Organization, vol. 86, no. 3, pp. 178-186, 2008. 